\title{
Por una escuela inclusiva: Reconocimiento de la diversidad escolar ${ }^{1}$
}

\author{
For an inclusive school: School diversity recognition
}

\author{
María Angélica Peñuela Rodríguez ${ }^{2}$ \\ Hugo Daniel Montoya Leiva ${ }^{3}$ \\ Aleida Fernández Moreno ${ }^{4}$
}

Recibido: Septiembre 162013 • Enviado para modificación: Noviembre 272013 • Aceptado: Diciembre 202013.

Peñuela, A.; Montoya, H.; Fernández, A. (2013). Por una escuela inclusiva: Reconocimiento de la diversidad escolar. Revista Ocupación Humana, 13 (2), pp. 24-39.

Resumen: Se busca valorar las condiciones inclusivas en el primer ciclo de dos instituciones educativas públicas de Bogotá y fomentar el conocimiento de los principios de la educación inclusiva como camino para garantizar la educación como derecho para todos. Usando el enfoque de Investigación acción participativa, se aplica el Índice de Inclusión adaptado por el Ministerio de Educación y se construyen proyecciones con las instituciones. Como resultados, se adapta el instrumento a cada plantel; la Institución 1 obtiene una puntuación de $2,4 / 4$, indicando que en ocasiones se realizan acciones inclusivas que son conocidas por la mayoría de los integrantes de la comunidad educativa; la Institución dos obtiene 3,5/4, mostrando una evaluación permanente para conocer el impacto de las acciones inclusivas en sus procesos administrativos y académicos, este resultado debe considerarse en el contexto de las características de esta escuela. El proceso permitió comprender la percepción de la comunidad sobre la inclusión, el aprendizaje en condiciones de igualdad, el desarrollo de oportunidades y el respeto por la diversidad; estos siguen manifestándose como dirigidos a poblaciones específicas. Las prácticas inclusivas que se desarrollan diariamente, son generadoras de oportunidades de aprendizaje, la mayor dificultad no es iniciarlas sino reconocer su potencial e importancia.

Palabras clave: Educación Inclusiva, igualdad de oportunidades, acceso a la educación

\begin{abstract}
The objective is to assess the inclusive conditions in two public educational institutions of Bogotá, and to promote the knowledge of inclusive education as a way to ensure education as a right for all, using the participatory action research approach. The assessment and projection tool used was the index for inclusion adapted by Ministerio de Educación Nacional. As a result, the instrument was adapted to each institution. Institution 1 was graded 2.4/4, indicating that in some occasions they implement inclusive actions known by most members of the school community. Institution 2 obtained 3.5/4, showing a permanent evaluation to determine the impact of inclusive actions in its administrative and academic processes; context of this school must be taken into account for this result. The process allowed us to understand the perception of the community about inclusion, equal learning, development opportunities

\footnotetext{
${ }^{1}$ Artículo basado en la tesis para optar al título de Terapeutas Ocupacionales de la Universidad Nacional de Colombia de Hugo Montoya y María Angélica Peñuela, dirigida por Aleida Fernández Moreno.

${ }^{2}$ Terapeuta Ocupacional. Secretaría Distrital de Integración Social. mapenuelar@unal.edu.co

${ }^{3}$ Terapeuta Ocupacional. Secretaría Distrital de Integración Social. hdmontoyal@unal.edu.co

${ }^{4}$ Terapeuta ocupacional. Magíster en Desarrollo Educativo y Social. Doctora en Ciencias Sociales, Niñez y Juventud. Coordinadora Maestría en Discapacidad e Inclusión Social. Universidad Nacional de Colombia. cafernandezm@unal.edu.co
} 
and regard for diversity; these are directed toward specific populations. Inclusive practices developed daily are generators of learning opportunities. The greatest difficulty is not to start them, but to recognize their potential and importance.

Keywords: Inclusive Education, equal opportunity, access to education.

Según la Comisión Económica para América Latina y el Caribe (CEPAL) y el Fondo de las Naciones Unidas para la Infancia (UNICEF) (CEPAL - UNICEF, 2010), la pobreza y otros elementos de la diversidad: niños y niñas afrodescendientes, diferencias de género y etnias, son factores que determinan el ingreso y la calidad de la educación básica. Estas organizaciones señalan que en América Latina hay un mayor número de personas que, por motivos relacionados directamente con su diversidad, no han asistido a instituciones educativas o han interrumpido sus estudios y agregan que las oportunidades de adquisición de conocimientos y competencias mínimas para la educación, se reducen principalmente por el acceso, la cultura, el ambiente, el equipamiento educativo y la formación de los docentes. Es por ello que la inclusión no se refiere exclusivamente a la condición de discapacidad, sino que incorpora otras diferencias. Ahora, esta problemática también se evidencia en Colombia, con las mismas disparidades en la educación. De acuerdo a la UNICEF (2013), en el país la cobertura bruta del primer ciclo alcanza apenas el $86 \%$ de los niños y las niñas de 5 y 6 años.

Para dar respuesta a estas situaciones, Colombia debe favorecer la educación de todos y todas las niñas en condiciones de calidad, acceso y oportunidad, acogiéndose así a los principios normativos que a nivel internacional y nacional la rigen. Entre ellos resalta la Convención sobre los derechos de las personas con discapacidad, aprobada por Colombia a través de la Ley 1346 de 2009 (Duarte y Osorno, 2013), la cual en su artículo 24 indica que "los Estados Partes reconocen el derecho de las personas con discapacidad a la educación con miras a hacer efectivo este derecho sin discriminación y sobre la base de la igualdad de oportunidades" (ONU, 2006). Adicionalmente en el contexto nacional, la Ley 115 de 1994 (Congreso de la República, 1994) prevé la educación para personas con limitaciones y con capacidades excepcionales, planteando que la educación de estos grupos es un servicio público de obligación para el Estado; recientemente la Ley 1618 de 2013 (Presidencia de la República, 2013), en su artículo 11, habla del derecho a la educación en las personas con necesidades educativas especiales (NEE), fomentando el acceso y la permanencia de las mismas en las instituciones públicas y privadas. Colombia ha asumido entonces el compromiso de generar estrategias que disminuyan los índices de deserción y las disparidades en la educación.

El enfoque actual de educación inclusiva, es producto de las transformaciones en la atención educativa de las personas con discapacidad en el mundo (ver Figura 1); esta en principio 
Figura 1. Cambios en la Educación: Ubicación temporal-espacial.

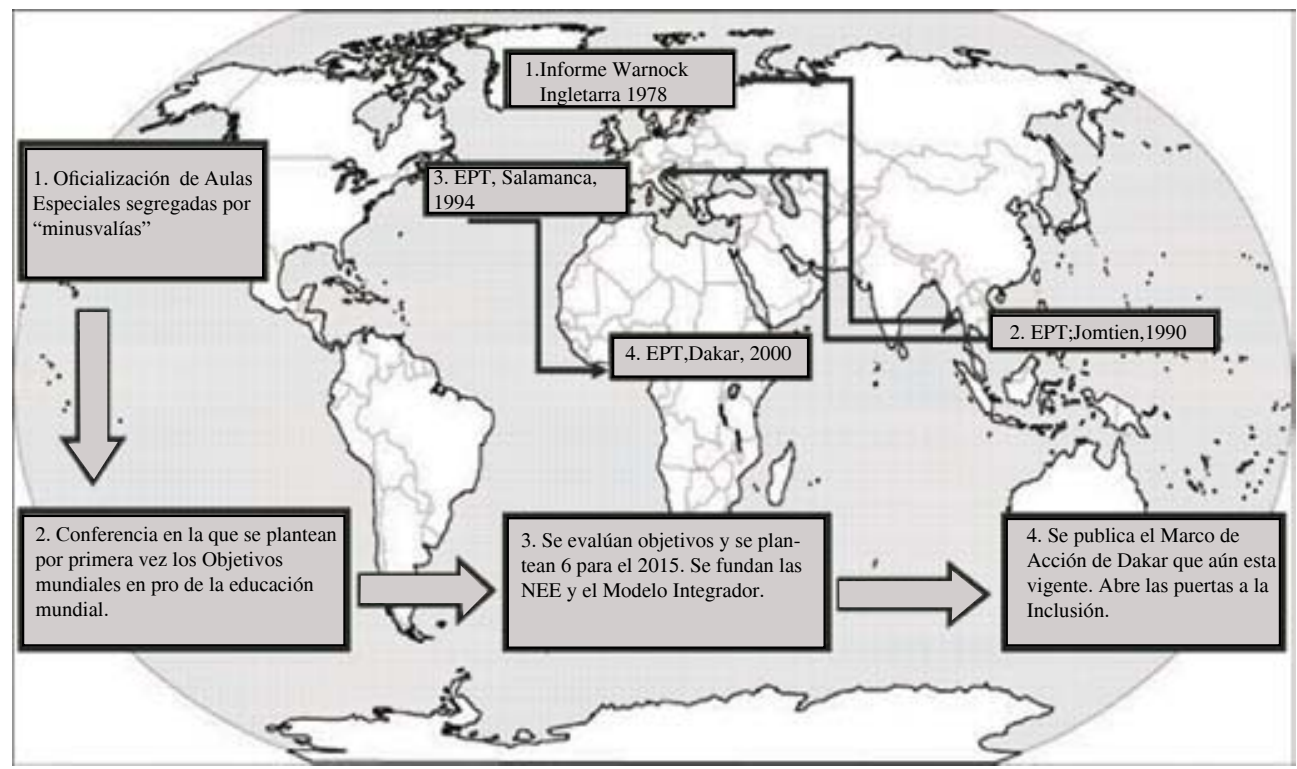

Fuente: Elaboración propia. Imagen de fondo: http://www.lacasainfantil.com

se enfocó en la discapacidad física, sensorial o mental, luego en las NEE, y en la actualidad en la atención a la diversidad, a continuación un breve recuento.

Tras décadas de educación especial en el mundo, en 1978 Mary Warnock presenta el que será reconocido como Informe Warnock, en donde se describen los trabajos realizados en pro de la educación hasta ese momento; allí se propone un modelo de integración escolar de la persona con discapacidad denominado Modelo de Educación Integradora (Warnock,1978).

Posteriormente, en 1990, se llevó a cabo en la ciudad de Jomtien (Tailandia) la Conferencia Mundial sobre Educación para Todos. En este evento se dieron a conocer las estrategias de participación educativa que se desa- rrollaban en los diferentes países y se propuso un marco de acción basado en diez objetivos, orientados a la satisfacción de las necesidades de aprendizaje por medio de la universalización de la educación básica; es allí donde surge el modelo de Educación Para Todos (EPT) (UNESCO, 1990).

Para 1994 en Salamanca, España, durante la Conferencia Mundial sobre Necesidades Educativas Especiales, se revisaron los avances en participación educativa de la población mundial hasta ese momento; en vista del limitado progreso, se optó por mantener solamente seis objetivos: 1 . Mejorar la atención de la primera infancia, 2. Enseñanza primaria gratuita y obligatoria para todos en 2015, 3. Acceso equitativo a los programas de preparación para la vida, 4. Lograr una mejora del $50 \%$ en la alfabetización de adultos 
para el año 2015, 5. Eliminación de las disparidades de género para 2005, y 6 . Lograr mejoras perceptibles en la calidad de la educación. Se planteó el acceso a las escuelas de las personas con NEE, con una pedagogía centrada en el niño y capaz de satisfacer esas necesidades (UNESCO, 1994).

Más adelante, en el año 2000, en el contexto del Foro Mundial sobre la Educación, surge el Marco de Acción de Dakar, el cual exige la educación como un derecho para niños, niñas, jóvenes y adultos. Lo más relevante, es la eliminación de disparidades de la infancia y un mayor énfasis en los niños y niñas con discapacidad, lo que promovió la integración de personas con discapacidad en las aulas regulares de escuelas que no ofrecían esa posibilidad (UNESCO, 2000).

Hoy día la atención educativa de las personas con discapacidad se orienta hacia la educación inclusiva, que con más de una década de existencia, ha avanzado en su paso de la teoría a la práctica; entonces, resulta importante ampliar este concepto.

La educación inclusiva actúa como:

un proceso que permite tener debidamente en cuenta la diversidad de las necesidades de todos los niños, jóvenes y adultos a través de una mayor participación en el aprendizaje, las actividades culturales y comunitarias, así como reducir la exclusión de la esfera de la enseñanza y dentro de ésta, y en último término acabar con ella. Entraña cambios y modificaciones de contenidos, enfoques, estructuras y estrategias basados en una visión común que abar- ca a todos los niños en edad escolar y la convicción de que corresponde al sistema educativo ordinario educar a todos los niños y niñas. (UNESCO, 2009, p.9)

Con base en el concepto de educación inclusiva y como resultado de las investigaciones realizadas en varias escuelas europeas, en el año 2000 los profesores Ainscow y Booth publicaron con la UNESCO el Índice de Inclusión (conocido como Índice de Inclusión de la UNESCO), un instrumento para la autoevaluación de las escuelas respecto de sus procesos de inclusión (Ainscow \& Booth, 2000).

Los cambios enunciados anteriormente, han generado impactos en la Terapia Ocupacional y en su ejercicio profesional en el área educativa. Según Álvarez (2010), a principios de los años setenta el terapeuta respondía a las necesidades particulares de niños "especiales" a través de la denominada atención pediátrica, las acciones se centraban en la rehabilitación bio-psicológica. Ya para finales de esa década, se empezaba a fomentar el trabajo desde el desempeño escolar de los niños hospitalizados; a partir de la ley de integración escolar, algunos centros empiezan a vincular terapeutas ocupacionales. En la década de los ochenta, cuando la participación del Terapeuta Ocupacional en la escuela era una realidad, se enfrentó a constantes planteamientos sobre la importancia de mejorar la calidad de la educación. La atención seguía siendo influenciada por el enfoque biomédiCo, el cual respondía al principio de atender a niños bajo el concepto de deficiencia o minusvalía; su intervención incidía directamente sobre las 
condiciones físicas, sensoriales y cognitivas de la población.

En el programa de Terapia Ocupacional de la Universidad Nacional de Colombia, se han realizado trabajos para abarcar el tema de la participación escolar que reflejan las transformaciones conceptuales. A finales de los años ochenta se desarrolló en el Instituto Pedagógico Nacional el Programa de Atención a las Necesidades Educativas Especiales en aulas regulares. Luego, a partir del 2000, en la localidad de Tunjuelito se implementó el Programa de Atención Integral al Escolar (PAIE), que incluía estrategias como los "rincones sensoriales", integración sensorial y el diálogo permanente con los padres de familia y los maestros para mejorar el desempeño ocupacional. Ya para los noventa la población atendida se hacía más diversa, dando respuesta a niños con dificultades de aprendizaje (Álvarez, 2010). En la actualidad, el llamado para la Terapia Ocupacional es a dar el paso hacia la educación inclusiva.

El objetivo principal del proyecto ES... inclusiva $^{5}$, en el cual se inserta el trabajo de grado que da origen a este artículo, es entonces valorar las condiciones inclusivas de dos instituciones educativas públicas de la ciudad de Bogotá, específicamente en el primer ciclo (preescolar, primero y segundo de primaria), y fomentar allí el conocimiento de los principios de la educación inclusiva como camino para garantizar la educación como derecho fundamental para todos.

\section{Metodología}

El estudio se basa en la revisión y aplicación del Índice de Inclusión de la UNESCO, en su versión adaptada para Colombia por el Ministerio de Educación Nacional (MEN) en 2009. El Índice de Inclusión es presentado por la UNESCO como una guía de evaluación que facilita a las escuelas obtener un indicador numérico del avance en los procesos inclusivos que llevan a cabo; sin embargo, sus autores insisten en que este no es solo un instrumento de valoración cuantitativa, sino una herramienta que da las pautas para desarrollar un plan de trabajo que conduzca a eliminar las barreras a la participación y el aprendizaje que enfrenta una comunidad particular.

Este estudio se plantea, en consecuencia, no como una evaluación, sino como parte de una estrategia de acompañamiento a las instituciones que se acerca a los planteamientos de la Investigación-acción participativa, una metodología que propone el análisis de la realidad como una forma de conocimiento a través de la intervención social (Basagoiti, Bru \& Lorenzana, 2001).

El proyecto tiene lugar en 2011 en dos instituciones educativas públicas de Bogotá ubicadas en la localidad de Engativá (en adelante, Institución 1 e Institución 2) ${ }^{6}$. La selección de las instituciones es apoyada por la Secretaria de Educación del Distrito, que facilita la conexión con las directivas de los

\footnotetext{
${ }^{5}$ Es....inclusiva es un proyecto de extensión de la Universidad Nacional de Colombia desarrollado en 2011 y en el marco del cual, entre otros productos, se elaboró el trabajo de grado del cual se deriva este artículo.

${ }^{6}$ Esta institución ofrece atención solamente a niños y niñas con deficiencia cognitiva, esta connotación hará que los resultados de su índice varíen considerablemente.
} 
colegios. El principal criterio de inclusión es el interés de las instituciones en revisar y avanzar en sus propios procesos de inclusión y en consecuencia, en aplicar el Índice.
Las comunidades educativas están conformadas por docentes, padres de familia, escolares y personal administrativo de la jornada tarde; el número de participantes se puede ver en la Tabla 1. De otra parte, el equipo de

Tabla 1. Participantes en el proyecto ES...inclusiva.

\begin{tabular}{|c|c|c|c|c|c|}
\hline \multirow{2}{*}{ Institución } & \multicolumn{3}{|c|}{ Número de participantes } & & Total \\
\hline & $\begin{array}{c}\text { Grupo Coordinador } \\
\text { (incluye administrativos) }\end{array}$ & Docentes & Padres de Familia & Estudiantes \\
\hline Institución 1 & 3 & 2 & 8 & 12 & 25 \\
\hline Institución 2 & 3 & 7 & 23 & 36 & 69 \\
\hline Total & & & 94 & & \\
\hline
\end{tabular}

Fuente: Montoya, H. \& Peñuela, M. (2012).

ES...inclusiva incluye terapeutas ocupacionales (profesionales y en formación), una psicóloga y una licenciada en Educación Física y Artes, cada uno de las cuales, desde su saber y habilidades, apoya el acompañamiento a las escuelas.
El índice de Inclusión de la UNESCO en su versión original comprende cinco etapas, teniendo en cuenta las limitaciones de tiempo y recursos para desarrollar este proyecto, se realizaron solo las tres primeras. El equipo de ES...inclusiva adaptó tales etapas y concretó el siguiente mapa de trabajo (Tabla 2):

Tabla 2. Etapas en el desarrollo del proyecto ES...inclusiva.

\begin{tabular}{|c|c|c|}
\hline$\sum_{\substack{\mid \\
\underbrace{}_{1}}}^{\infty}$ & \multicolumn{2}{|c|}{$\begin{array}{l}\text { 1. Surgimiento de ES...inclusiva } \\
\text { 2. Constitución del equipo de trabajo } \\
\text { 3. Selección de instituciones educativas } \\
\text { 4. Planeación de estrategias } \\
\text { 5. Revisión comparada del Índice de Inclusión Unesco y el Índice de Inclusión MEN y adapta- } \\
\text { ción del instrumento para el estudio. }\end{array}$} \\
\hline \multirow{5}{*}{  } & \multicolumn{2}{|c|}{ 1. Concertación de las instituciones educativas } \\
\hline & \multirow{3}{*}{$\begin{array}{l}\text { 2. Planeación y elabo- } \\
\text { ración de estrategias }\end{array}$} & Comunicativas: Folletos, textos facilitadores, cartillas ilustrativas. \\
\hline & & Visuales: Cartel interactivo, tarjetas, guías. \\
\hline & & $\begin{array}{l}\text { Participativas: Talleres con padres, docentes, y ciclo I (Actividades de } \\
\text { sensibilización, cartografías de la diversidad, cuadernillos de inclusión, } \\
\text { construcción de planes de acción). }\end{array}$ \\
\hline & \multicolumn{2}{|c|}{$\begin{array}{l}\text { 3. Desarrollo de talleres con cada comunidad educativa. Aplicación de cuestionarios del Índice a } \\
\text { docentes, padres de familia y alumnado. }\end{array}$} \\
\hline$\sum_{\substack{\infty \\
c}}^{\infty}$ & \multicolumn{2}{|c|}{$\begin{array}{l}\text { 1. Tabulación y análisis de los resultados de los cuestionarios. } \\
\text { 2. Interpretación de los rangos cuantitativos. } \\
\text { 3. Presentación de resultados y construcción concertada de planes de mejoramiento. } \\
\text { 4. Elaboración de informes técnicos por cada Institución educativa. }\end{array}$} \\
\hline
\end{tabular}

Fuente: Montoya, H. \& Peñuela, M. (2012). 
El índice adaptado para Colombia por el MEN logra entrelazar las necesidades generales de la población colombiana y brindar algunas estrategias que faciliten el proceso inclusivo. Según el MEN (2009) "el Índice es una herramienta que permite a la institución educativa realizar el proceso de autoevaluación de la gestión inclusiva, reconociendo el estado actual en la atención a la diversidad, el análisis de fortalezas y oportunidades de mejoramiento" (p. 12); mide la gestión de las condiciones de aprendizaje, participación y convivencia de todos sus estudiantes desde cuatro áreas: directiva, administrativa, académica y comunidad (Figura 2), las cuales se consideran los ejes de desarrollo para cualificar la gestión de la inclusión educativa.

Figura 2. Áreas de valoración del Índice de Inclusión versión MEN (2009).

\begin{tabular}{|l|}
\hline \multicolumn{1}{|c|}{ Área Académica } \\
\hline - Diseño pedagógico \\
(curricular). \\
- Prácticas \\
pedagógicas. \\
- Gestión de aula. \\
- Seguimiento \\
académico. \\
\\
\end{tabular}

\begin{tabular}{|l|}
\hline Área Administrativa \\
\hline - Apoyo a la gestión \\
académica. \\
- Administración de la \\
planta física y de los \\
recursos. \\
- Administración de \\
servicios comple- \\
mentarios. \\
- Talento humano. \\
- Apoyo financiero y \\
contable.
\end{tabular}

Fuente: Montoya, H. \&Peñuela, M. (2012).

El instrumento comprende la metodología de aplicación y los tres cuestionarios que se aplican a los padres de familia, los profesores y los alumnos. Cada cuestionario incluye un listado de acciones y prácticas frente a las cuales se asignan valoraciones de acuerdo a la frecuencia con que ocurren en la escuela (4: siempre, 3: casi siempre, 2: algunas veces, 1: no sé, 0 : no se hace). Posterior a la aplicación, se promedian los resultados por cada una de las áreas de gestión. Luego se realiza la interpretación a partir de los rangos de puntajes (Tabla 3). (Ver página siguiente).

Finalmente, después de presentar a las comunidades los puntajes obtenidos



\begin{tabular}{|l|}
\hline \multicolumn{1}{|c|}{ Área Directiva } \\
\hline - Direccionamiento estra- \\
tégico y horizonte insti- \\
tucional. \\
- Gerencia estratégica. \\
- Gobierno escolar. \\
- Cultura institucional. \\
- Clima escolar. \\
- Relaciones con el \\
entorno. \\
\end{tabular}

en cada área de gestión y su interpretación, se usan como punto de partida para elaborar los planes de mejoramiento para cada escuela, a través de talleres participativos.

\section{Resultados}

Se presentarán en el siguiente orden: Adaptación del instrumento, reflexiones a partir de los talleres de la Etapa 2, calificaciones obtenidas a partir de la aplicación del Índice de InclusiónMEN y planes de mejoramiento.

\section{Comparación y adaptación del Instrumento:}

La comparación entre los dos índices (UNESCO y MEN) muestra, en tér- 
Tabla 3. Bases para la interpretación de resultados.

\begin{tabular}{|l|l|}
\hline Rangos & Interpretación \\
\hline $0.0-0.99$ & $\begin{array}{l}\text { Los resultados en este rango indican que no se desarrollan acciones inclusivas } \\
\text { para la atención a la diversidad en el proceso o área de gestión. }\end{array}$ \\
\hline $1.0-1.99$ & $\begin{array}{l}\text { Los resultados en este rango indican que la comunidad educativa desconoce las } \\
\text { acciones inclusivas que desarrolla la institución para la atención a la diversidad } \\
\text { en el proceso o área de gestión. }\end{array}$ \\
\hline 2.0-2.79 & $\begin{array}{l}\text { Los resultados en este rango indican que en algunas ocasiones se realizan accio- } \\
\text { nes inclusivas para la atención a la diversidad en el área de gestión propia y son } \\
\text { conocidas por la mayoría de los integrantes de la comunidad educativa. }\end{array}$ \\
\hline $2.8-3.49$ & $\begin{array}{l}\text { Los resultados en este rango indican que con frecuencia se realizan acciones } \\
\text { inclusivas para la atención a la diversidad en el proceso o área de gestión y son } \\
\text { conocidas por todos los integrantes de la comunidad educativa. }\end{array}$ \\
\hline $3.5-4.0$ & $\begin{array}{l}\text { Los resultados en este rango indican una evaluación permanente para conocer el } \\
\text { impacto de las acciones inclusivas para la atención a la diversidad en el proceso } \\
\text { o área de gestión que se usa para aportar al desarrollo institucional. }\end{array}$ \\
\hline
\end{tabular}

Fuente: MEN, 2009.

minos de aproximación a las diversidades (etnias, composición familiar, género, discapacidad y desplazamiento $^{7}$ ), lo siguiente:

Etnias: Aun cuando en Colombia el $10.6 \%$ de la población se autoreconoce como afrocolombiana (DANE, 2005), el Índice adaptado por el MEN no cuenta con preguntas que hagan referencia a ese grupo, los indicadores se centran principalmente en la población con discapacidad o en situación de vulnerabilidad, específicamente desplazamiento forzado. Entre tanto, algunas de la preguntas del Índice dela UNESCO, le apuestan a la multiculturalidad.

Composición familiar: El índice de UNESCO brinda herramientas para involucrar a los miembros de la familia en el contexto curricular y en las dinámicas pedagógicas que se construyen

${ }^{7}$ Estás categorías fueron seleccionadas por el equipo de ES...inclusiva. día a día con los niños, niñas y jóvenes en procesos de formación académica, la adaptación colombiana sesga esta categoría.

Género: La versión colombiana, a diferencia de la de UNESCO, en ninguna de sus preguntas habla sobre las diversidades de género, tampoco plantea actividades o cuestionamientos para resaltarlas.

Discapacidad: Este es uno de los ítems que mayor énfasis tiene en todos los indicadores de las dos versiones del Índice; las preguntas se relacionan con las dificultades que puedan enfrentar los niños y niñas en situación de discapacidad física, sensorial o mental, al igual que los posibles recursos y prácticas pedagógicas que se desarrollan para dinamizar procesos de aprendizaje y adaptación.

Desplazamiento: Aunque esta es una de las situaciones que la versión co 
lombiana relaciona con la condición de vulnerabilidad, no se le da mayor énfasis; el número de preguntas dirigidas a identificar acciones que promuevan el respeto y la valoración de esta población es el mismo que dedica la UNESCO, cuatro.

Adicionalmente, el índice versión para Colombia adapta el vocabulario de los indicadores para la población del país, haciéndolos más comprensibles para nuestro contexto. Sin embargo, el equipo de ES...inclusiva no está de acuerdo en usar el término Necesidades Educativas Especiales, en tanto este suele relacionarse directamente con la población con discapacidad, en detrimento del concepto de diversidad al que apunta la educación inclusiva, y centra la atención en el individuo y no en el contexto, ni en la relación entre ambos. En compensación, en el proyecto se retoma el término "Barreras para la participación y el aprendizaje", que usa la versión original de la UNESCO.

\section{Talleres con maestros:}

Los talleres propician espacios de reflexión donde se comparten las vivencias personales y colectivas dentro de la escuela. Al inicio, los docentes están expectantes, pues según ellos varias de las experiencias previas en temas relacionados habían sido negativas y poco productivas. Los talleres permiten explorar la percepción de los docentes frente al tema de la diversidad: En la institución 1 había resistencia y comentarios negativos al respecto, "la inclusión nos ha costado trabajo", "no estamos capacitados para atender a más de tres niños con discapacidades"; por el contrario en la institución 2, los comentarios eran positivos: "aquí en la institución recibimos a los niños que no ingresan en otras instituciones por sus condiciones", "nuestro trabajo se basa en las capacidades y no en las dificultades".

Con el paso de los talleres, los docentes amplían su concepción de la diversidad y exploran características diferentes a la discapacidad; por esta razón, en el segundo y el tercer taller el énfasis de los debates se centra en la atención a la diversidad.

\section{Calificaciones obtenidas a partir de la aplicación del Índice de Inclusión:}

La gráfica 1 muestra los resultados obtenidos en cada área de gestión ${ }^{8}$ :

Para la institución 1, las áreas que mostraron calificaciones más altas fueron la académica y la directiva, lo cual indica que las acciones inclusivas se realizan con frecuencia, aquí se resalta la capacitación a la población y la revisión de la accesibilidad de los espacios. Entre tanto, en las áreas administrativa y de comunidad son pocas las acciones que se realizan, principalmente se evidencia la escasa participación de las familias y otros miembros de la comunidad en la institución; estas áreas requieren especial atención en los procesos de reflexión y planeación institucional, de manera que las propuestas de acción relacionadas promuevan un mayor desarrollo.

\footnotetext{
${ }^{8}$ Con las gráficas no se pretende realizar una comparación entre las dos instituciones, solamente muestra los puntajes obtenidos en cada área valorada.
} 
Figura 3. Resultados por áreas del Índice de Inclusión del MEN.
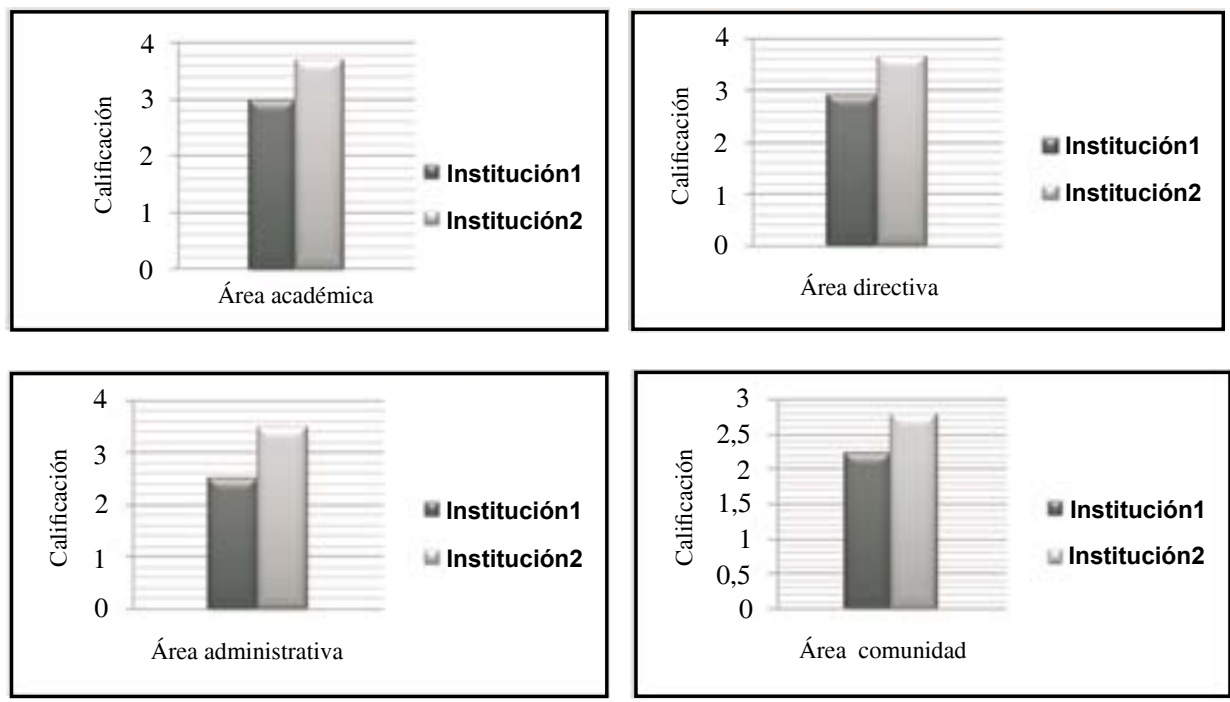

Fuente: Montoya, H. \& Peñuela, M. (2012).

Para la institución 2, el área más potente fue la académica, en la cual resaltan indicadores como la revisión y mejoramiento permanente de los planes de estudio y el enfoque metodológico; continúa con el área administrativa, evidenciando los esfuerzos en flexibilización curricular; luego aparece comunidad, con la planeación de proyectos de vida para estudiantes y familias; finalmente se encuentra el área directiva, donde se resalta la participación en la construcción de una política pública de discapacidad a nivel local.

El Índice de Inclusión de la institución 1 es $2,4 / 4$, lo que indica que en algunas ocasiones se realizan acciones inclusivas y son conocidas por la mayoría de los integrantes de la comunidad educativa.

El Índice de Inclusión de la institución 2 es 3,5/4, que indica una evaluación permanente para conocer el impacto de las acciones inclusivas. Sin embargo, no se puede afirmar que esta es una institución inclusiva, dado que la definición de UNESCO promueve la participación de todos los niños y niñas de la comunidad dentro de un ambiente escolar regular. En este caso, la calificación obedece a las adaptaciones que la institución hizo al cuestionario, omitiendo la premisa: acceso a toda la comunidad; así, si bien sus prácticas favorecen los procesos de aprendizaje, no así la participación de poblaciones diversas, pues la institución admite solo estudiantes con limitación cognitiva.

\section{Construcción de planes de mejoramiento:}

A partir de los resultados, cada institución elaboró con sus recursos las proyecciones más relevantes. En la tabla 4 , se presentan algunas de las pro 
puestas de cada institución para las diferentes áreas del Índice de Inclusión: Las propuestas se centran en la planeación y la realización de acciones que favorezcan las condiciones físicas y de accesibilidad de la institución, la flexibilización curricular, los procesos de sensibilización y el apoyo a y con las familias. Las instituciones empiezan a generar cambios en el camino hacia la educación inclusiva: El primer cambio que propone la institución 1 es fortalecer la gestión académica flexibilizando el currículo con nuevas metodologías, acercando a los niños con discapacidad a la escuela durante uno o dos días cada semana hasta que logre adaptarse completamente. La institución 2, propone acciones en el área directiva, específicamente en lo relacionado con la gestión para la adecuación de la planta física.

Con miras a proyectar un mayor impacto en la localidad, el proyecto ES... inclusiva realizó una reunión con el propósito de presentar los resultados obtenidos a los representantes de la

Tabla 4. Propuestas de mejoramiento más relevantes.

\begin{tabular}{|c|c|c|}
\hline & Institución 2 & Institución 1 \\
\hline & $\begin{array}{l}\text { - Realizar evaluación de la planta física del } \\
\text { colegio a principio del año escolar. } \\
\text { - Construir un modelo pedagógico flexible para } \\
\text { el desarrollo humano a través de la educación } \\
\text { formal. } \\
\text { - Fortalecer el comité de evaluación y promo- } \\
\text { ción. }\end{array}$ & $\begin{array}{l}\text { - Adecuación real de la planta física y la infra- } \\
\text { estructura en general. } \\
\text { - Solicitar apoyos pedagógicos desde fonoaudio- } \\
\text { logía y diferentes escuelas de terapias a través } \\
\text { de prácticas académicas. } \\
\text { - Reevaluar el PEI y la concepción de "niños de } \\
\text { inclusión" en el manual de convivencia. }\end{array}$ \\
\hline & $\begin{array}{l}\text { - Desarrollar estrategias metodológicas y cam- } \\
\text { bios curriculares para que se ajusten a las nece- } \\
\text { sidades de los estudiantes. } \\
\text { - Crear relaciones interculturales en los ciclos } \\
\text { intra-institucional y extra-institucional. }\end{array}$ & $\begin{array}{l}\text { - Realizar flexibilización curricular y diversifica- } \\
\text { ción curricular. } \\
\text { - Establecer convenios que permitan el acceso } \\
\text { a todos los servicios que en salud se puedan } \\
\text { ofrecer para establecer la condición del niño de } \\
\text { forma eficaz. }\end{array}$ \\
\hline & $\begin{array}{l}\text { - Elaborar los programas de apoyo familia a fa- } \\
\text { milia. } \\
\text { - En el fortalecimiento de redes: Intercambio de } \\
\text { recursos y servicios con otros colegios públi- } \\
\text { cos de la localidad y de la cuidad. } \\
\text { - Generar acciones específicas para la preven- } \\
\text { ción de riesgos y la atención de emergencias. }\end{array}$ & $\begin{array}{l}\text { - Buscar apoyo en los padres de familia para la } \\
\text { colaboración en el aula. } \\
\text { - Sensibilizar a toda la comunidad educativa so- } \\
\text { bre la inclusión y sus diferencias con la inte- } \\
\text { gración. } \\
\text { - Enfatizar dentro del trabajo cultural lo ético en } \\
\text { cuanto a valores de la diversidad. }\end{array}$ \\
\hline 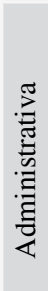 & $\begin{array}{l}\text { - Continuar con las acciones de sensibilización } \\
\text { que orientan a los participantes en la compren- } \\
\text { sión y el respeto por las diferencias del ser hu- } \\
\text { mano. } \\
\text { - Aportar a la formación y capacitación en la } \\
\text { educación de niños y niñas con discapacidad } \\
\text { cognitiva para el personal de otros colegios pú- } \\
\text { blicos de la localidad. }\end{array}$ & $\begin{array}{l}\text { - Incluir estas acciones en los planes de emergen- } \\
\text { cia institucionales. } \\
\text { - Incluir en la planeación y ejecución de las ac- } \\
\text { ciones tendientes a la inclusión, mecanismos de } \\
\text { seguimiento y realimentación continúa a partir } \\
\text { de resultados intermedios y finales DE INCLU- } \\
\text { SIÓN que trasciendan indicadores de rendi- } \\
\text { miento académico. }\end{array}$ \\
\hline
\end{tabular}

Fuente: Montoya, H. \& Peñuela, M. (2012). 
Secretaria de Educación del Distrito (SED), la Secretaria Distrital de Integración y la Secretaria Distrital de Salud. Las entidades no conocían algunas de las necesidades de las instituciones, se comprometieron entonces a brindar programas y acciones para apoyarlas. Aun cuando los representantes de la SED no pudieron asistir a este encuentro, los resultados y sugerencias se le enviaron por escrito.

En el desarrollo del proyecto, Es...inclusiva elaboró algunos productos de utilidad para las comunidades participantes, pero también para proyectos futuros con otras instituciones interesadas, estos son:

Informes finales. Son los productos más potentes del proyecto, en estos se presenta el análisis cuantitativo y cualitativo de los resultados de la aplicación del Índice Inclusión y de los talleres realizados con padres, niños y docentes y finalizan con el planteamiento de las acciones a desarrollar en cada una de las instituciones. Estos informes pueden ser consultados en el repositorio de investigaciones en discapacidad disponible en el siguiente enlace: http://www.coloquiodiscapacidad.com/investigaciones/index.php/ investigaciones

Blog Es...inclusiva. Constituye una iniciativa diseñada para aportar a los colegios información útil para aumentar su conocimiento y mejorar sus procesos de inclusión. Si bien requiere ser alimentado y actualizado, resulta una fuente de información abierta y con gran potencial. La dirección web es: http://esinclusiva.blogspot.com/

Cartilla Ilustrada (Figura 4). Es una herramienta de comunicación del proyecto ES...inclusiva a las comunidades educativas en la que, por medio de frases e ilustraciones, se hace referencia a la educación inclusiva como una oportunidad de participación y reconocimiento de la diversidad en la escuela. Fue ilustrada por el profesor de la Facultad de Artes de la Universidad Nacional, Carlos Martin Riaño.

Figura 4. Fotografías de la Cartilla Ilustrada.
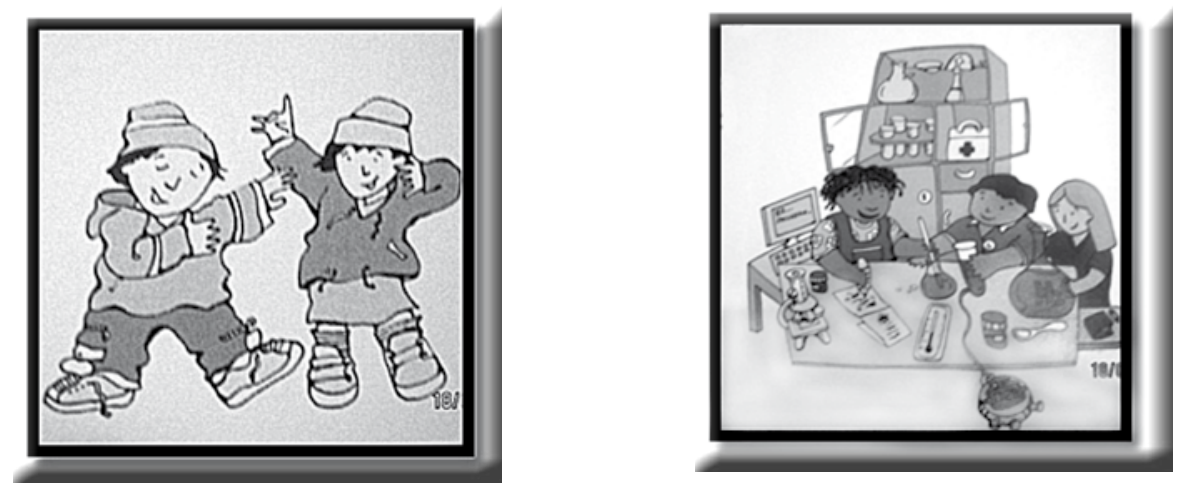


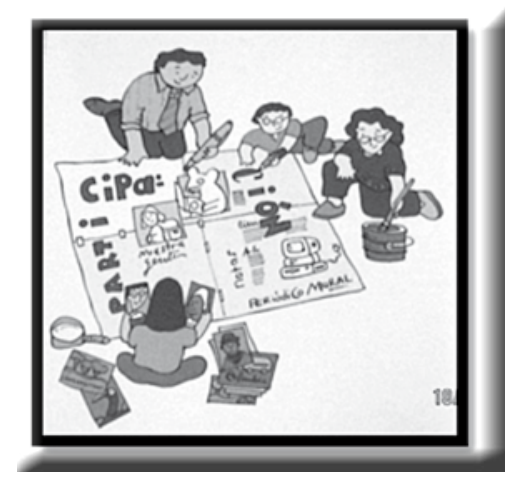

Fuente: Fotografías tomadas de la cartilla original.

\section{Discusión}

El proyecto ES... inclusiva valora la importancia de analizar y utilizar instrumentos que, como el Indice de Inclusión, son guías fundamentales para el ejercicio en equipos interdisciplinarios en instituciones educativas. Esta experiencia permite evidenciar los cambios favorables que se pueden dar a partir de intervenciones cortas pero concienzudas y fundamentadas en resultados cuantitativos y cualitativos.

Aunque el MEN (2010) refiere haber aplicado el instrumento en más de 33 instituciones en Colombia, no se encuentran registros de ello, por lo cual identificar y analizar experiencias de aplicación es una tarea difícil. Esta deficiencia en la difusión de los resultados de su aplicación, además desdibuja, en alguna medida, el papel que realiza la entidad en el tema de educación inclusiva. No obstante, es importante agregar que en la actualidad el MEN adelanta la validación del instrumento, los resultados de este ejercicio se proyectan para 2014.

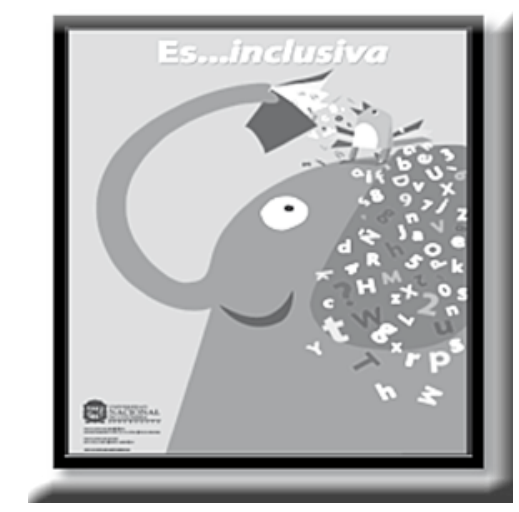

Solo se encontró documentada la experiencia de una institución ubicada en Funza. Según la Gobernación de Cundinamarca (2011), en esta institución las puntaciones en el índice de inclusión varían entre 2,6 y 3.0, lo cual la identifica como una institución que involucra algunos procesos de inclusión. Una de las similitudes que se puede identificar entre las aplicaciones de ES... Inclusiva y la de este colegio, es que buena parte de las fortalezas se centran en las gestiones de tipo directivo y administrativo; la escasez de prácticas en el área académica, muestran las deficiencias de la gestión de los recursos físicos y humanos en ese aspecto. Por otro lado, hay similitudes en el área de gestión con la comunidad, donde difícilmente se involucra a la familia y a otros entes externos que podrían apoyar los procesos de inclusión en las escuelas.

Lo anterior puede ser entendido como una tendencia de las instituciones a centrar la gestión en el nivel directivo, sin participación de la comunidad amplia. Al respecto el profesor Ainscow, 
en una entrevista concedida en la Universidad de Deust, indica que "en los países pobres, el foco está en construir escuelas, dar materiales y formar a profesores porque hay 17 millones de niños que no tienen una escuela donde ir. Pero en países ricos como España o el Reino Unido, donde tenemos suficientes escuelas y profesores, el sistema sigue fallando en muchos sentidos" (Ainscow, 2011). Es importante reconocer entonces que la inclusión no es el fin, es el medio para disminuir las disparidades en las escuelas y que facilita la capacidad para responder a la diversidad.

\section{Conclusiones}

Este ejercicio permitió identificar que el Índice de Inclusión del MEN es un instrumento que presenta algunos vacíos. En primera instancia, los cuestionarios no responden a la diversidad de la población Colombiana; de otra parte, la metodología de trabajo que plantea no se compara con la del Î́ndice de la UNESCO, ya que reduce gran cantidad de actividades previas a la aplicación de los cuestionarios que son vitales para la comprensión de la diversidad.

Los talleres generaron aportes importantes para las comunidades educativas, se reconoció que además de las discapacidades físicas, cognitivas y sensoriales que presentan varios de sus estudiantes, hay otras condiciones o situaciones (género, edad, raza, cultura, condiciones económicas, ser víctima del desplazamiento forzado, entre otras que expresan la diversidad presente en la escuela) que hacen el llamado a ser tenidas en cuenta, como las discapacidades, en los planes de mejoramiento.

Los resultados del Índice evidencian que las instituciones necesitan hacer esfuerzos importantes para transformar las culturas y políticas, de manera que favorezcan la inclusión, el respeto y la valoración de la diversidad. Un tema esencial y prioritario es la accesibilidad en términos de tecnología, modificaciones ambientales y recurso humano.

A partir de los resultados cuantitativos es posible afirmar que para lograr la inclusión se requieren transformaciones en la comunidad (padres, cuidadores, vecinos, entre otros), generando su participación y compromiso con la educación de los niños. Estas transformaciones deben iniciar por el respeto a la diversidad, el cambio de pedagogías dentro del aula, una labor sustentada en el desarrollo de capacidades y una evaluación ponderada de acuerdo a las diferencias que caractericen a cada estudiante, constante evaluación de los procesos de formación, transformaciones arquitectónicas, flexibilización curricular, acompañamiento a estudiantes, promoción del uso de tecnología, informática y otras herramientas, como estrategias para eliminar barreras para el aprendizaje.

Finalmente, el rol del Terapeuta Ocupacional viene dando un giro oportuno para la profesión, afianzando los conocimientos desde el ámbito educativo y fortaleciendo procesos de mayor compromiso con las instituciones educativas. Esta sin duda es una invitación a todos aquellos terapeutas que ejercen en el campo de la de la edu 
cación, para que logren movilizar sus intervenciones desde el enfoque biomédico, centrado en las deficiencias, hacia los procesos de aprendizaje que propone la educación inclusiva.

\section{Agradecimientos}

El proyecto Es... Inclusiva pudo desarrollarse gracias a la financiación de la Facultad de Medicina de la Universidad Nacional de Colombia y al soporte de los miembros del Grupo de Investigación Discapacidad, inclusión y Sociedad, de la Universidad Nacional de Colombia.

\section{Referencias}

Ainscow, M. \& Booth, T. (2000) Índice de inclusión de la UNESCO. (A. López \& R. Blanco, Trad.) Recuperado de: http:// www.eenet.org.uk.

Ainscow, M. (2011, 24 de Noviembre). La inclusión no es acerca de grupos concretos de estudiantes, es reestructurar el sistema educativo. Euskadi+innova. Recuperado de: http://www.euskadinnova. net/.

Álvarez, L. (2010). Terapia Ocupacional en educación: Un enfoque sensorial en la escuela. Bogotá: Universidad Nacional de Colombia.

Basagoiti, M.; Bru, P. \& Lorenzana, C. (2001). IAP (de bolsillo): Investigaciónacción participativa. Madrid: Acsur.

CEPAL - UNICEF. (2010). Pobreza Infantil en América Latina y el Caribe. Recuperado de: http://www.unicef.org/lac/ Libro-pobreza-infantil-America-Latina-2010\%281\%29.pdf.

Congreso de la República de Colombia (1994). Ley 115 de 1994: Ley General de Educación. Recuperado de: http:// www.mineducacion.gov.co/1621/articles-85906_archivo_pdf.

Departamento Administrativo Nacional de Estadística - DANE-. (2005). Censo general 2005. Recuperado de: http:// www.dane.gov.co/censo/files/libroCenso2005nacional.pdf.

Duarte, C.; Osorno, M. (2013). Desarrollo de la legislación en discapacidad en Colombia. Revista Ocupación Humana, 13 (1), pp. 33-53.

Gobernación de Cundinamarca. (2011). Proyecto de educación inclusiva en Cundinamarca: Resultados de aplicación del Índice de Inclusión, Institución Educativa Departamental Miguel Antonio caro. Recuperado de: https://docs. google.com/document/d/1Y5m65WLX9 bMVNSUn2s6oAtpjl6nMPb3TwrrK1eB Re0g/edit.

Ministerio de Educación Nacional. (2009). Programa de educación inclusiva con calidad "Construyendo capacidad institucional para la atención a la diversidad": Índice de Inclusión. Recuperado de: http://www.colombiaaprende.edu.co/ html/micrositios/1752/articles-320693_ Pdf_1.pdf.

Montoya, H. \& Peñuela, A. (2012). Por una escuela Inclusiva: Reconocimiento de la diversidad escolar. Universidad Nacional de Colombia, Bogotá.

Organización de las Naciones Unidas ONU. (2006). Convención sobre los derechos de las personas con discapacidad. Recuperado de: http://www.un.org/spanish/disabilities/default.asp?id=497.

Presidencia de la República. (2013). Ley Estatutaria 1618 de 2013. Recuperado de: http://wsp.presidencia.gov.co/Normativa/Leyes/Documents/2013/LEY\%20 1618\%20DEL\%2027\%20DE\%20FEBRERO\%20DE\%202013.pdf.

UNESCO. (1990). Meeting basic learning needs: A vision for the 1990s. Recupe- 
rado de: http://unesdoc.unesco.org/ images/0009/000975/097552e.pdf.

UNESCO. (1994). Declaración de Salamanca y Marco de Acción para las Necesidades Educativas Especiales. Recuperado de: http://www.unesco.org/education/pdf/ SALAMA_S.PDF.

UNESCO. (2000). Marco de Acción de Dakar - Educación para Todos: Cumplir nuestros compromisos comunes. Recuperado de: http://unesdoc.unesco.org/ images/0012/001211/121147s.pdf.
UNESCO. (2009). Directrices sobre políticas de inclusión en la educación. Recuperado de: http://unesdoc.unesco.org/ images/0017/001778/177849s.pdf.

UNICEF. (2013). ¿Cómo están las niñas y los niños en Colombia?. Recuperado de: http://www.unicef.com.co/situacion-dela-infancia/.

Warnock, M. (1978). Special educational needs: Report of the Committee of enquiry into the education of handicapped children and young people. London. 


\section{Guía abreviada para autores}

Antes de enviar un artículo a la Revista Ocupación Humana, los autores deben asegurarse de haber leído la "Guía de Autores" completa, disponible en la página http://www.tocolombia.org/revistaoh/. Los artículos o documentos deben enviarse al correo electrónico editorial@tocolombia.org, adjuntando la carta remisoria con los nombres y firmas del autor o autores, especificando sus correspondientes datos de identificación (nombre, dirección, dirección electrónica y teléfonos). Se anexará además una carta con la certificación de responsabilidad del (los) autor(es), la cesión de los derechos de publicación y la declaración de conflictos de interés. Una vez recibido el artículo y tras la verificación del cumplimiento de los requisitos, la Editora hace su presentación ante los Comités Editorial y Científico, quienes evalúan su mérito científico. Posteriormente el artículo será sometido a evaluación por pares, quienes revisarán y aprobarán su contenido y podrán realizar observaciones sobre el mismo.

La Revista considera la publicación de documentos de los siguientes tipos: Artículos de Investigación, de reflexión, de revisión, reportes de caso, revisiones de tema, cartas al editor, reseñas bibliográficas, artículos cortos y revisiones temáticas.

Los textos presentados reunirán las siguientes características: Elaboración en tamaño carta, letra Arial, tamaño 12, interlineado 1.15, y referencias bibliográficas en letra tamaño 10. La extensión máxima será de 15 páginas, las cuales deben ir numeradas. Debe incluirse un resumen en español e inglés, entre 150 y 200 palabras, con sus respectivas palabras clave (máximo cinco) de acuerdo al índice de Tesauro de la Unesco, los Descriptores en Ciencias de la Salud DeCS y $\mathrm{MeSH}$. Es responsabilidad de los autores asegurar la calidad de la traducción presentada.

Las tablas y figuras deben estar referenciados en el texto y contener numeración y título, si ya han sido publicadas, se debe mencionar la fuente. Si el artículo cuenta con fotografías, estas deben ser de alta resolución y calidad; si se trata de imágenes de usuarios o pacientes, se debe anexar el respectivo consentimiento para su publicación.

Las figuras y tablas serán enviados en archivos separados del texto, en formato Word para Windows; deben ser legibles, presentados en blanco y negro o en escala de grises. Se deben seguir las normas de la American Psychological Association (APA), en su última versión publicada.

Las referencias bibliográficas se organizarán en orden alfabético según el primer apellido del autor y se ubicarán en una hoja aparte al final del artículo, con la estructura que se presenta a continuación:

Trujillo, A. (2002). Terapia ocupacional: conocimiento y práctica en Colombia. Bogotá: Editorial Universidad Nacional de Colombia.

Otro tipo de citación (revistas, artículos electrónicos, entre otros) será consultada por los autores en la norma APA.

\section{Suscripción a la Revista}

Los profesionales y estudiantes colegiados reciben en su dirección de correspondencia en Colombia y sin costo alguno, los dos números anuales de la Revista.

Para los interesados no colegiados los costos son:

- Valor unitario: $\$ 20.000$

- Suscripción anual para residentes en Colombia (dos números + gastos de envío a nivel nacional): $\$ 50.000$.

- Suscripción anual para residentes fuera del país: $\$ 50.000$ + gastos de envío (tarifa vigente al momento del envío).

Para suscribirse a la Revista Ocupación Humana diligencie el formato y envíelo por correo postal a la Cra. 9B \# 117 A 15, Bogotá - Colombia, o escaneado al correo electrónico editorial@tocolombia.org

Formato de suscripción:

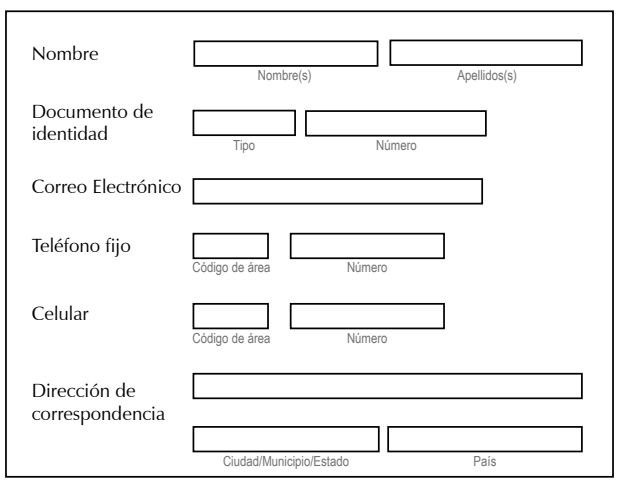

